\title{
Left-right Polynomial Matrix Factorization for MIMO Pole/Zero Cancellation with Application to FEL*
}

\author{
Kenji SugImoto $^{\dagger}$ and Wataru ImahaYASHI ${ }^{\dagger}$
}

\begin{abstract}
Motivated by a biological motion control model called feedback error learning (FEL), feedforward learning control schemes have been extensively studied with emphasis on their implementation by linear filters and tuning parameters. Its multi-input multi-output (MIMO) generalization, however, has been redundant in terms of pole/zero cancellation structure. The objective of this paper is to propose an exact feedforward controller by introducing the notion of left-right polynomial matrix factorization.
\end{abstract}

\section{Introduction}

Pole/zero cancellation plays an important role in various two-degrees-of-freedom (2DOF) control system design schemes such as model reference adaptive control and feedback error learning (FEL). The former is further generalized to VRFT scheme $[1,2]$. In the latter, fixed feedback (FB) controller stabilizes the closed-loop while feedforward (FF) controller is tuned on-line to attain a good response by canceling the stable zeros of the plant; see [3-6], just to name a few. This "role-sharing" in 2DOF structure is promising in practice since we can concentrate $\mathrm{FB}$ control on robust stability, while tuning FF control gives much better transient property than other adaptive control schemes that also require stabilization.

Application of FEL to mechanical systems can be found say in [7-9], but they have been confined to single-input single-output (SISO) plant so far. The authors' group have tried to generalize this scheme to the multi-input multi-output (MIMO) case [10-12]. Most of these works, however, resorted to a certain shortcut to address non-commutativity of matrix multiplication, and hence suffers from a redundancy.

The objective of this paper is to refine the MIMO tuning FF controller by introducing the novel concept of left-right factorization by polynomial matri-

\footnotetext{
* Manuscript Received Date: June 29, 2018

The material of this paper was partially presented at the 49th ISCIE International Symposium on Stochastic Systems Theory and Its Applications (SSS'17) which was held in Nov, 2017.

† Graduate School of Science and Technology, Nara Institute of Science and Technology; 8916-5 Ikoma, Nara 630-0192, JAPAN
}

Key Words: parameter estimation, system modeling, two-degrees-of-freedom, feedforward control, polynomial matrix. ces, thereby giving an exact parameterization for pole/ zero cancellation. It is interesting to note that left and right factorizations of polynomial matrices have been extensively used for representing MIMO systems $[13,14]$, but left-right matrix factorization has never been used, though its inverse form appeared half a century ago [15].

This paper is organized as follows. After mentioning preliminaries in Section 2, we discuss parametric issues of polynomial matrices in Subsection $\mathbf{3 . 1}$ and attain pole/zero cancellation in Subsection 3.2. Subsection $\mathbf{3 . 3}$ is devoted to some examples. Then we develop a tuning law for FF controller in Subsection 3.4. This is done by a recursive least square algorithm. Numerical simulation is carried out in Section 4 to show the effectiveness of the proposed method.

Notation: We often use upper cases for polynomial matrices, and lower cases with a single (double) subscript for their row vectors (for their entries, respectively); e.g.,

$$
N(s)=\left(\begin{array}{c}
n_{1}(s) \\
\vdots \\
n_{p}(s)
\end{array}\right)=\left(\begin{array}{ccc}
n_{11}(s) & \cdots & n_{1 p}(s) \\
\vdots & \ddots & \vdots \\
n_{p 1}(s) & \cdots & n_{p p}(s)
\end{array}\right) .
$$

We further add superscript for their coefficients; e.g., $n_{i j}(s)=\sum_{k=0}^{\mu} n_{i j}^{k} s^{\mu-k}$, where $n_{i j}^{k}$ does not mean the $k$ th power of $n_{i j}$. $I_{p}$ denotes the $p$-th order identity but we may omit the suffix when the size is irrelevant or obvious. $\mathbb{N}$ and $\mathbb{C}$ respectively denote the set of positive integers and the set of complex numbers.

All signals are described in the frequency domain up to Section 3.2. In Section 3.4 time domain signals are also treated since we tune the parameter in FF controller on-line. 


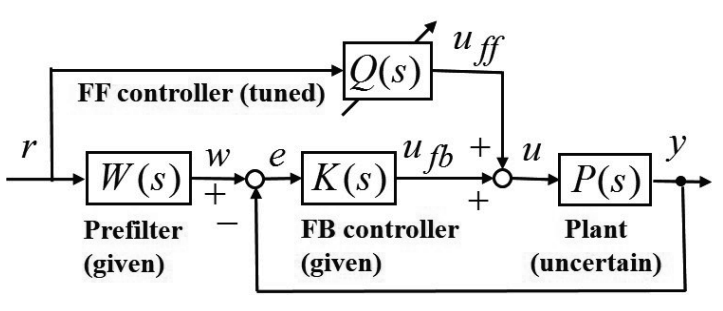

Fig. 1 2DOF structure with tuning FF controller

\section{Preliminaries}

\subsection{Control Objective}

Consider the configuration of Fig. 1, where $P(s)$ is a plant to be controlled, whose coefficients are uncertain. Assume that FB controller $K(s)$ stabilizes the closed-loop, either by a model-free design such as PID control or a robust control design, but it gives a poor response to the reference signal $r$. We improve the response by tuning FF controller $Q(s)$ rather than by $K(s)$, thereby avoiding the conflict between sensitivity reduction and robust stability, which we often confront in usual FB control design.

To be more specific, let us assume that $P(s)$ is a $p \times p$ strictly proper rational matrix of minimum phase; i.e., $\operatorname{det} P(s) \neq 0$ for all $s \in \mathbb{C}$ such that $\operatorname{Re} s \geq 0$. We further assume that

$$
\operatorname{det} E \neq 0, E:=\left(\begin{array}{c}
c_{1} A^{\rho_{1}-1} B \\
\vdots \\
c_{p} A^{\rho_{p}-1} B
\end{array}\right), C=\left(\begin{array}{c}
c_{1} \\
\vdots \\
c_{p}
\end{array}\right),
$$

where $(A, B, C)$ is a minimal realization of $P(s)$, and

$$
\rho_{i}:=\min \left\{\rho \in \mathbb{N} ; c_{i} A^{\rho-1} B \neq 0\right\}, \quad i=1, \cdots, p .
$$

We call $\rho_{i}$ the $i$-th row relative degree. Then we can take a polynomial matrix

$$
\begin{aligned}
& L(s)=\operatorname{diag}\left(\ell_{1}(s), \cdots, \ell_{p}(s)\right), \\
& \ell_{j}(s)=\ell_{j}^{0} s^{\rho_{j}}+\cdots+\ell_{j}^{\rho_{j}-1} s+1, \quad j=1, \cdots, p,
\end{aligned}
$$

where $\ell_{j}(s)$ are Hurwitz polynomials. In adaptive control literature $L(s)$ is called interactor. It is easily verified that $L(s) P(s)$ is a biproper rational matrix.

Now we can formally state our control objective as follows. Take $W(s)=L(s)^{-1}$, which can be regarded as a response model. (Taking (2) requires that the signals are decoupled and the steady gain is unity for each output.) We want the feedback error $e=w-y$ to vanish, where $w=W(s) r$. In this paper we attain this by tuning FF controller $Q(s)$ so that

$$
P(s) Q(s)=W(s)
$$

\subsection{Discussion}

Since $W(s)$ is zero-free, (3) means that the zeros of $P(s)$ must be canceled by the poles of $Q(s)$. For the SISO case it is easy to take a biproper function

$$
Q(s)=\frac{d(s)}{n(s) \ell(s)}, \quad \text { where } \quad P(s)=\frac{n(s)}{d(s)},
$$

so that

$$
P(s) Q(s)=\frac{n(s)}{d(s)} \cdot \frac{d(s)}{n(s) \ell(s)}=\frac{1}{\ell(s)} .
$$

Our objective is to give a natural MIMO extension of the above SISO pole/zero cancellation, which is by no means trivial.

As is well known $[13,14]$, a left (or right, respectively) coprime factorization by polynomial matrices is given by

$$
P(s)=D(s)^{-1} N(s) \quad\left(=\tilde{N}(s) \tilde{D}(s)^{-1},\right)
$$

where $(D, N)(\operatorname{or}(\tilde{N}, \tilde{D}))$ is a left (or right) coprime pair, namely

$$
\operatorname{rank}(D(s) N(s))=p \quad\left(\operatorname{rank}\left(\begin{array}{c}
\tilde{D}(s) \\
\tilde{N}(s)
\end{array}\right)=p\right)
$$

for all $s \in \mathbb{C}$. Then we can write

$$
D(s) y=N(s) u,
$$

where $u$ and $y$ are input and output vector signals of the plant respectively. It is important to note that in i/o representation (6), the coefficients appear linearly, hence the left, rather than right, factorization is suitable for on-line estimation; see Section $\mathbf{3 . 4}$ for a detail.

From (4) it is obvious that

$$
Q(s)=N(s)^{-1} D(s) L(s)^{-1}
$$

is biproper and satisfies (3). We call (7) left-right factorization in this paper. This is, however, not easy to directly implement as FF controller since matrix multiplication is noncommutative and we cannot pack $N(s)$ and $L(s)$ into one factor. In the configuration of Fig. 1, taking (7) is equivalent to

$$
N(s) u_{f f}=D(s) w,
$$

but this is not directly realizable, because $P(s)^{-1}=$ $N(s)^{-1} D(s)$ is improper. We have to implement the three matrices in (7) in a way compatible with parameter tuning.

\section{$2.3 \quad$ Existing Schemes}

The authors' group proposed a method for the MIMO pole/zero cancellation $[10,11]$ as follows. Write

$$
P(s)^{-1}=M(s)+\frac{1}{g(s)} R(s),
$$

where $g(s)$ is the least common multiple denominator polynomial of the entries of $P(s)^{-1}$, Each entries of $M(s)$ and $\frac{1}{g(s)} R(s)$ are respectively the polynomial part and the strictly proper part of the corresponding entry of $P(s)^{-1}$. Then

$$
Q(s)=\{g(s) I\}^{-1}\{g(s) M(s)+R(s)\} L(s)^{-1}
$$




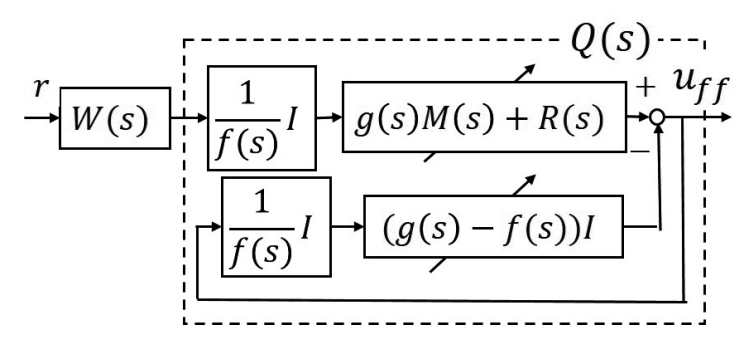

Fig. 2 Conventional scheme for FF controller

attains (3) and can be realizable by linear filters. We further transform this into

$$
Q(s)=\left(\frac{g(s)}{f(s)}\right)^{-1}\{g(s) M(s)+R(s)\}\{f(s) L(s)\}^{-1},
$$

where we take a Hurwitz polynomial $f(s)$ having the same degree of $g(s)$. In (9), plant-dependent parameters appear linearly and are tuned on-line (Fig. 2). The tuned blocks are improper in itself but they can be realized by using the state variables in $L(s)^{-1}$ and $f(s)^{-1} I$; see $[10,11]$ for a detail. They thus avoid the difficulty pointed out in Subsection 2.2 via scalar polynomials $f(s), g(s)$. A drawback of $(9)$ is that it suffers from an over-parameterization and hence tuning may be insufficient; see Sections $\mathbf{3 . 3}$ and $\mathbf{4}$.

Another scheme has been proposed in [12], where the numerator matrix was parameterized to address MIMO pole/zero cancellation. This is, however, implemented by using multiple filters for each of the signal channels in their approach, which is also too redundant.

\section{Main Result}

\subsection{Parametric Structure}

It is widely known that left coprime factorization (4) is not unique; see, e.g., [13,14]. We often assume that denominator matrix $D(s)$ is row proper [13], where its row degrees agree with the observability indices of $(A, B, C)$. In this paper, however, we are more interested in numerator matrix $N(s)$, thus we first introduce the following notion.

[Definition 1] If a polynomial matrix is written with its row vectors

$$
N(s)=\left(\begin{array}{c}
n_{1}(s) \\
\vdots \\
n_{p}(s)
\end{array}\right), \quad n_{i}(s)=\sum_{k=0}^{\mu_{i}} n_{i}^{k} s^{\mu_{i}-k}
$$

where $n_{i}^{0} \neq 0$ for $i=1, \cdots, p$, then $\mu_{i}$ is called the $i$-th row degree of $N(s)$. Furthermore, define the constant matrices

$$
[N(s)]_{r}:=\left(\begin{array}{c}
n_{1}^{0} \\
\vdots \\
n_{p}^{0}
\end{array}\right) \text { and }[N(s)]_{c}:=\left(\left[N(s)^{\mathrm{T}}\right]_{r}\right)^{\mathrm{T}} .
$$

Namely, the matrix $[N(s)]_{r}$ (or $[N(s)]_{c}$, respectively) consists of the highest-degree coefficient row (column) vectors.

[Lemma 1] Let $P(s)$ be a square rational function matrix that is invertible and strictly proper. Then it admits left coprime factorization (4) such that

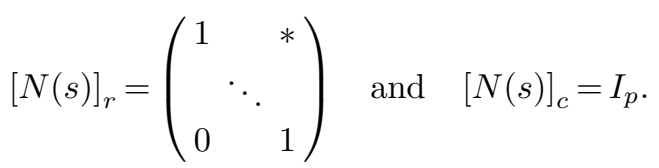

(Proof) It is known [13] that there exists left coprime factorization (4) such that $N(s)$ is row proper (also called row reduced by [14]); namely,

$$
\operatorname{det}[N(s)]_{r} \neq 0 \text {. }
$$

It is also known that $(\bar{D}, \bar{N})$ gives another left coprime factorization iff

$$
\bar{D}(s)=U(s) D(s), \quad \bar{N}(s)=U(s) N(s)
$$

for any unimodular polynomial matrix $U(s)$, and that premultiplying the pair by such $U(s)$ is equivalent to applying a series of row transformation to it; see $[13,14]$.

Let us derive the coprime factorization satisfying (11) by such transformations. The first condition has been already shown; see the Appendix of [12]. Furthermore, we can derive a pair such that the row degrees of $N(s)$ are non-increasing; i.e., $\mu_{1} \geq \mu_{2} \geq \cdots \geq$ $\mu_{p}$. Then it is straightforward to show that row transformations lead to the second condition of (11), along with the first condition. This completes the proof.

It is easy to show that in the above numerator matrix

$$
\operatorname{deg} \operatorname{det} N(s)=\sum_{i=1}^{p} \mu_{i}
$$

which is equal to the number of zeros of $P(s)$. Furthermore, the degrees of the denominator matrix are characterized by the following lemma.

[Lemma 2] Given (4), (11), and the interactor (2), the denominator matrix is parameterized as

$$
D(s)=\left(d_{i j}(s)\right), \quad d_{i j}(s)=\sum_{k=0}^{\mu_{i}+\rho_{j}} d_{i j}^{k} s^{\mu_{i}+\rho_{j}-k}
$$

and $\operatorname{det}\left(d_{i j}^{0}\right) \neq 0$ holds.

(Proof) By the definition of interactor

$$
P(s)^{-1} L(s)^{-1}=N(s)^{-1} D(s) L(s)^{-1}
$$

is biproper. Furthermore,

$$
\operatorname{diag}\left(s^{\mu_{1}}, \cdots, s^{\mu_{p}}\right)^{-1} N(s), L(s) \operatorname{diag}\left(s^{\rho_{1}}, \cdots, s^{\rho_{p}}\right)^{-1}
$$

is also biproper. Hence

$$
\Xi(s)=\operatorname{diag}\left(s^{\mu_{1}}, \cdots, s^{\mu_{p}}\right)^{-1} D(s) \operatorname{diag}\left(s^{\rho_{1}}, \cdots, s^{\rho_{p}}\right)^{-1}
$$

is biproper. We have $\Xi(\infty)=\left(d_{i j}^{0}\right)$, which is nonsin- 


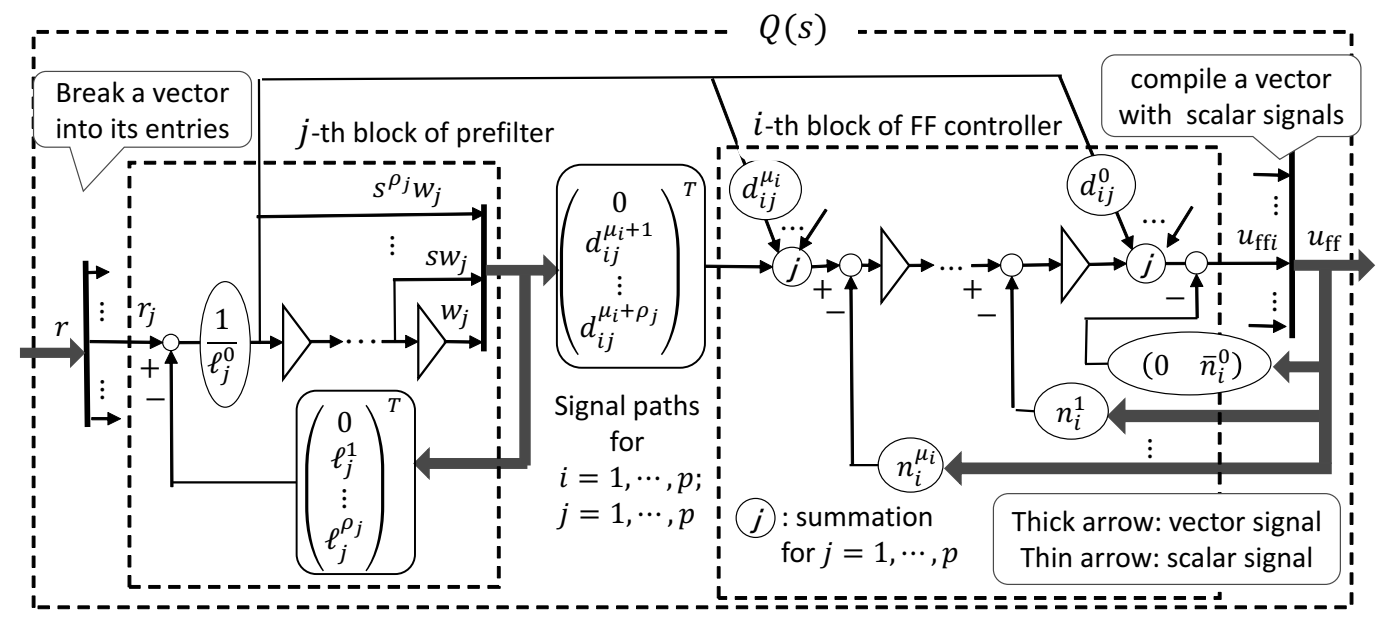

Fig. 3 Block diagram of FF controller $Q(s)$ for MIMO pole/zero cancellation

gular. This completes the proof.

(Remark 1) Lemma 2 prescribes the number of coefficients in the denominator matrix. But $d_{i j}^{0} \neq 0$ does not necessarily hold for all $i, j$; i.e., $d_{i j}^{0}=0$ may be true for some $i, j$.

\subsection{Pole/Zero Cancellation}

Let us rewrite (8) in terms of coefficients of each row:

$$
\begin{aligned}
& \left(\sum_{k=0}^{\mu_{i}} n_{i}^{k} s^{\mu_{i}-k}\right) u_{f f}=\sum_{j=1}^{p}\left(\sum_{k=0}^{\mu_{i}+\rho_{j}} d_{i j}^{k} s^{\mu_{i}+\rho_{j}-k}\right) w_{j} \\
& i=1, \cdots, p \text {. }
\end{aligned}
$$

From (11), the highest-degree coefficient vector of $N(s)$ is written as

$$
n_{i}^{0}=\left(\begin{array}{lllll}
0 & \cdots & 0 & 1 & \bar{n}_{i}^{0}
\end{array}\right)
$$

where $\bar{n}_{i}^{0}$ is a $(p-i)$-dimensional row vector.

Now we have the following theorem.

[Theorem 1] The FF controller $Q(s)$ satisfying (12) is implemented by the block diagram given by Fig. 3 .

(Proof) By tracing back from the right end of the diagram, for $i=1, \cdots, p$, we can easily show

$$
\xi_{i 1}=s\left(n_{i}^{0} u_{f f}-\sum_{j=1}^{p} d_{i j}^{0} s^{\rho_{j}} w_{j}\right)
$$

by (13). Proceeding similarly to the left, we finally obtain

$$
\begin{aligned}
\xi_{i \mu_{i}} & =s\left(\xi_{i \mu_{i}-1}-\sum_{j=1}^{p} d_{i j}^{\mu_{i}-1} s^{\rho_{j}} w_{j}+n_{i}^{\mu_{i}-1} u_{f f}\right) \\
& =\cdots \\
& =\sum_{k=1}^{\mu_{i}}\left(n_{i}^{\mu_{i}-k} s^{k} u_{f f}-\sum_{j=1}^{p} d_{i j}^{\mu_{i}-k} s^{\rho_{j}+k} w_{j}\right) .
\end{aligned}
$$

On the other hand, in view of the adding point at the left side of $\xi_{i \mu_{i}}$, we have

$$
\xi_{i \mu_{i}}=-n_{i}^{\mu_{i}} u_{f f}+\sum_{j=1}^{p} \sum_{k=0}^{\rho_{j}} d_{i j}^{\mu_{i}+k} s^{\rho_{j}-k} w_{j} .
$$

Finally we obtain (12) by equating (14) and (15).

\subsection{Examples}

\subsubsection{Uniform Degree Case}

We first consider the so-called uniform degree case:

$$
\rho:=\rho_{1}=\cdots=\rho_{p}, \quad \mu:=\mu_{1}=\cdots=\mu_{p} .
$$

In this case, we can write the interactor (4) and left fraction (2) as

$$
\begin{aligned}
L(s) & =\sum_{k=0}^{\rho} L_{k} s^{\rho-k}, \quad L_{\rho}=I_{p} . \\
D(s) & =\sum_{k=0}^{\mu+\rho} D_{k} s^{\mu+\rho-k}, N(s)=I_{p} s^{\mu}+\sum_{k=1}^{\mu} N_{k} s^{\mu-k} .
\end{aligned}
$$

The above equations contain plant-dependent matrices $D_{k}, N_{k}$ hence the number of parameters is $\# Q=$ $(2 \mu+\rho+1) p^{2}$. For brevity let $\mu=2$, and $\rho=2$. Then $\# Q=7 p^{2}$ and the FF controller (7) is implemented by the block diagram in Fig. 4. The structure turns out to be rather simple in this case, but it is interesting to see that the feedback loop is separated into two parts that implement the interactor $L(s)$ and the numerator matrix $N(s)$ respectively.

In the conventional scheme [10], we take $g(s)=$ $\operatorname{det} N(s)$ whose degree is $p \mu$ and entries of $g(s) M(s)+$ $R(s)$ have degree $p \mu+\rho+1$. Hence The total number is $\# Q=p \mu+(p \mu+\rho+1) p^{2}$. In the above case we have $\# Q=2 p^{3}+3 p^{2}+2 p$, which becomes large if $p>1$.

\subsubsection{Non-uniform Degree Case}

We then treat the non-uniform degree case. Typically, let $p=2$ and $\left(\rho_{1}, \rho_{2}\right)=(1,2),\left(\mu_{1}, \mu_{2}\right)=(1,0)$. Take the interactor and numerator polynomial matrices

$$
L(s)=\left(\begin{array}{cc}
s+1 & 0 \\
0 & \left(\frac{1}{2} s+1\right)^{2}
\end{array}\right), \quad N(s)=\left(\begin{array}{cc}
s+n_{11}^{1} & 0 \\
0 & 1
\end{array}\right)
$$




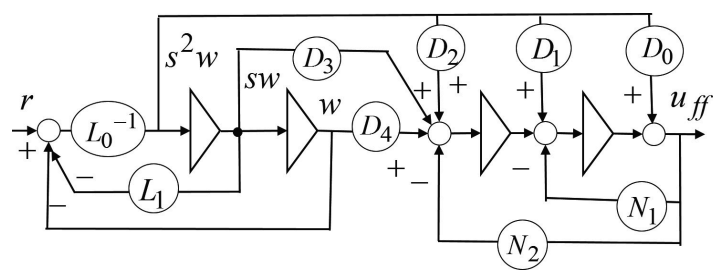

Fig. 4 The uniform degree case (all lines indicate vector signals)

as an example. Note that this system has one zero. It often happens that two-input two-output systems have an odd number of zeros as in this case, hence non-uniform degrees are by no means a rare case.

By Theorem 1, we have the denominator matrix

$$
\begin{gathered}
D(s)=\left(\begin{array}{cc}
d_{11}^{0} s^{2}+d_{11}^{1} s+d_{11}^{2} & d_{12}^{0} s^{3}+\cdots+d_{12}^{3} \\
d_{21}^{0} s+d_{21} & d_{22}^{0} s^{2}+d_{22}^{1} s+d_{22}^{2}
\end{array}\right), \\
\operatorname{det}\left(\begin{array}{ll}
d_{11}^{0} & d_{12}^{0} \\
d_{21}^{0} & d_{22}^{0}
\end{array}\right) \neq 0
\end{gathered}
$$

in general. Hence FF controller for pole/zero cancellation is implemented by the block diagram in Fig. 5 .

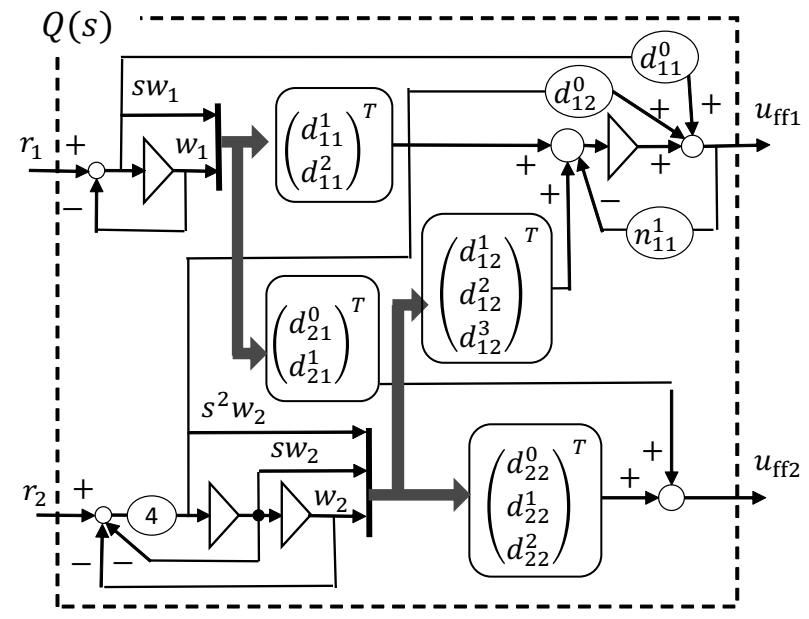

Fig. 5 The non-uniform degree case

Let us check that $u_{f f}=Q(s) r$ by this diagram satisfies (8). We first note that the output signal in the upper-right integrator is given by

$$
\xi=u_{f f 1}-d_{11}^{0} s w_{1}-d_{12}^{0} s^{2} w_{2},
$$

but the input of the same integrator is

$$
\begin{aligned}
s \xi= & -n_{11}^{1} u_{f f 1}+d_{11}^{1} s w_{1}+d_{11}^{2} w_{1} \\
& +d_{12}^{1} s^{2} w_{2}+d_{12}^{2} s w_{2}+d_{12}^{3} w_{2} .
\end{aligned}
$$

By equating these, we obtain the first row of (8). The second row is also obtained in a similar way.

We can calculate $\# Q=13$ in the above case, while in the conventional scheme $[10]$ we have $\# Q=15$.

\subsection{Parameter Tuning}

In this subsection we tune the parameter in Fig. 3 so that pole/zero cancellation is achieved even if the plant parameters are unknown. This is equivalent to on-line identification of the coefficients in the i/o relation (6). We start by rewriting this as

$$
\left(n_{i 1}(s), \cdots, n_{i p}(s)\right) u=\left(d_{i 1}(s), \cdots, d_{i p}(s)\right) y
$$

for each row. Then we take Hurwitz polynomial $\tilde{d}_{i}(s)$ such that

$$
\operatorname{deg} \tilde{d}_{i}(s)=\nu_{i}:=\mu_{i}+\max _{j} \rho_{j}
$$

and divide the both sides of $(16)$ by $\tilde{d}_{i}(s)$ to obtain relationship between signals generated by proper transfer matrices:

$$
\begin{gathered}
\frac{s^{\mu_{i}}}{\tilde{d}_{i}(s)} u_{i}=\theta_{i} V_{i}(s)\left(\begin{array}{c}
u \\
y
\end{array}\right) \\
\theta_{i}=\left(-\bar{n}_{i}^{0},-n_{i}^{1}, \cdots,-n_{i}^{\mu_{i}}\right. \\
\left.d_{i 1}^{0}, \cdots, d_{i 1}^{\mu_{i}+\rho_{1}}, \cdots, d_{i p}^{0}, \cdots, d_{i p}^{\mu_{i}+\rho_{p}}\right) \\
V_{i}(s)=\frac{1}{\tilde{d}_{i}(s)} \text { block-diag }\left\{\left(\begin{array}{c}
s^{\mu_{i}}\left[0, I_{p-i}\right] \\
s^{\mu_{i}-1} I_{p} \\
\vdots \\
I_{p}
\end{array}\right),\right. \\
\left.\left(\begin{array}{c}
s^{\mu_{i}+\rho_{1}} \\
\vdots \\
1
\end{array}\right), \cdots,\left(\begin{array}{c}
s^{\mu_{i}+\rho_{p}} \\
\vdots \\
1
\end{array}\right)\right\}
\end{gathered}
$$

As in $[10,11]$, we can tune the parameter $\theta_{i}$ on-line by taking the error model

$$
\begin{aligned}
& \varepsilon_{i}=\hat{u}_{i}-\theta_{i} \xi_{i} \rightarrow 0, \\
& \hat{u}_{i}=\frac{s^{\mu_{i}}}{\tilde{d}_{i}(s)} u_{i}, \quad \xi_{i}=V_{i}(s)\left(\begin{array}{c}
u \\
y
\end{array}\right) .
\end{aligned}
$$

A typical tuning law is given by

$$
\begin{aligned}
\dot{\theta}_{i}(t) & =\varepsilon_{i}(t) \xi_{i}(t)^{\mathrm{T}} Z_{i}(t), \\
\dot{Z}_{i}(t) & =-Z_{i}(t) \xi_{i}(t) \xi_{i}(t)^{\mathrm{T}} Z_{i}(t), \\
\theta_{i}(0) & =0, \quad Z_{i}(0)=\sigma I, \quad \sigma \gg 1 .
\end{aligned}
$$

This is a kind of recursive least square algorithm in continuous-time.

\section{Numerical Simulation}

Numerical simulation has been carried out for the denominator and numerator matrices in Section 3.3.2, with specific values as:

$$
\begin{aligned}
& D(s)=\left(\begin{array}{cc}
6 s^{2}+4 s+4 & 3 s^{3}+4 s^{2}+4 \\
s & 5 s^{2}+6 s+7
\end{array}\right), \\
& N(s)=\left(\begin{array}{cc}
s+2 & 0 \\
0 & 1
\end{array}\right), \quad K(s)=\left(\begin{array}{cc}
1+\frac{1}{s} & 0 \\
0 & 1+\frac{1}{s}
\end{array}\right) .
\end{aligned}
$$

Stability of the closed-loop has been checked. We apply rectangular waves of period $T=10[\mathrm{~s}]$ and unity magnitude as $r_{1}$ and $r_{2}$ with a phase delay of $5[\mathrm{~s}]$ in the latter:

$$
r_{1}(t)= \begin{cases}1 ; & 0 \leq t<5 \\ -1 ; & 5 \leq t<10\end{cases}
$$


which is extended periodically, and

$$
r_{2}(t)= \begin{cases}0 ; & 0 \leq t<5 \\ r_{1}(t-5) & 5 \leq t\end{cases}
$$

In the tuning law (19), we take $\sigma=10000$.

Figs. 6 through 7 are simulation results for the proposed scheme. In an early stage the output is insufficient but after tuning we obtain perfect tracking (Fig. 6). Fig. 7 shows time evolution of the tuned parameter.

Figs. 8 through 10 are simulation results for the conventional scheme [10]; see (9) and Fig. 2. We observe that tuning does not work well and even after some time tracking is insufficient (Figs. 8 and 9). Parameter tuning does not converge either, at least during this period (Fig. 10).

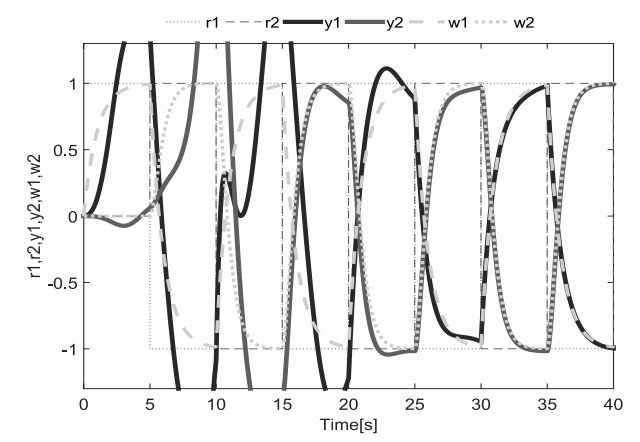

Fig. 6 Tracking by proposed scheme; $0[\mathrm{~s}] \sim 40[\mathrm{~s}]$

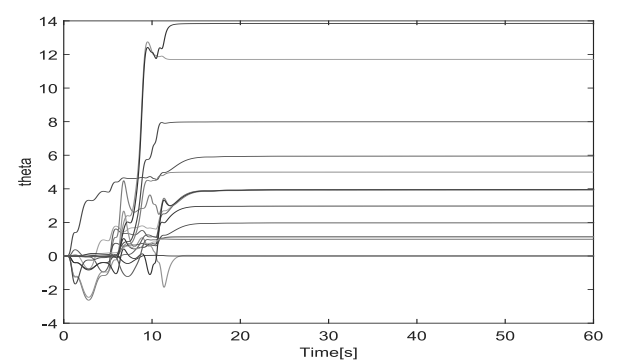

Fig. 7 Time evolution of estimated parameter by proposed scheme

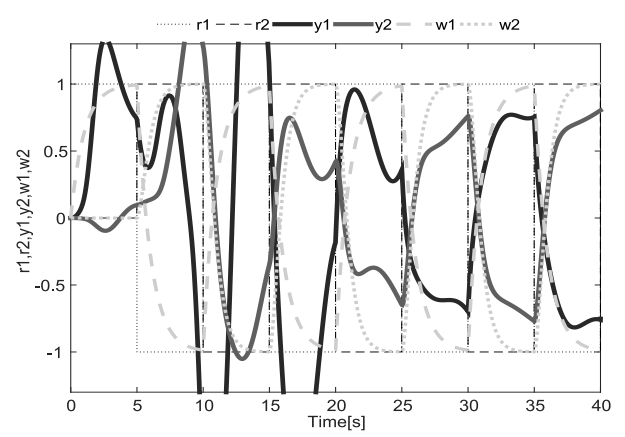

Fig. 8 Tracking by conventional scheme; $0[\mathrm{~s}] \sim 40[\mathrm{~s}]$

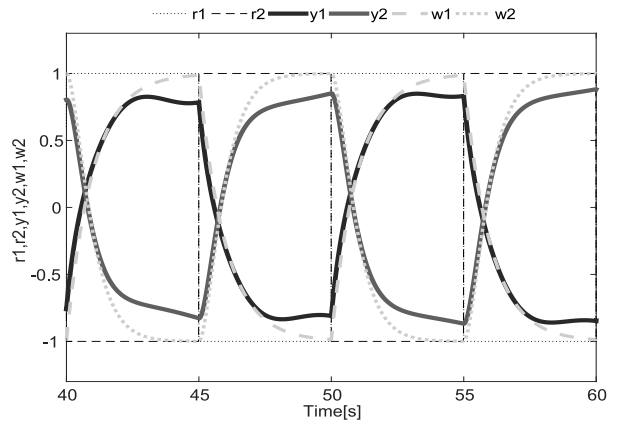

Fig. 9 Tracking by conventional scheme; $40[\mathrm{~s}] \sim 60[\mathrm{~s}]$

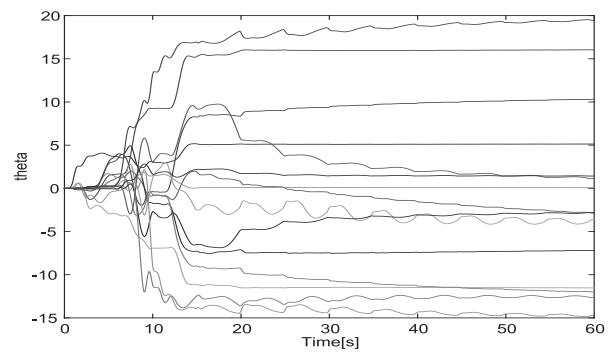

Fig. 10 Time evolution of estimated parameter by conventional scheme

\section{Acknowledgements}

The authors would like to thank Assistant Professor Masaki Ogura for his helpful comments and graduate student Xinyou Han for his assistance. This work was supported in part by JSPS Kakenhi under the grant \# JP $15 \mathrm{~K} 06141$.

\section{References}

[1] S. Masuda, X. Kong, K. Udagawa and Y. Matsui: Virtual reference feedback tuning for two degree of freedom controllers using closed-loop step response data-optimal pre-filter design in the frequency domain; 54th Annual Conference of the Society of Instrument and Control Engineers (SICE 2015), pp. 622-625 (2015)

[2] O. Kaneko, Y. K. Beak and T. Ohtsuka: Closed loop identification based on virtual reference feedback tuning applied to a virtual two-degree-of-freedom control system; SICE Journal of Control Measurement and System Integration, Vol. 2, No. 2, pp. 168176 (2009)

[3] M. Kawato, K. Furukawa and R. Suzuki: A hierarchical neural-network model for control and learning of voluntary movement; Biol. Cybern., Vol. 57, No. 3, pp. $169-185$ (1987)

[4] A. Miyamura and H. Kimura: Stability of feedback error learning scheme; Systems \& Control Letters, Vol. 45, No. 4, pp. 303-316 (2002)

[5] E. Muramatsu and K. Watanabe: Feedback error learning control without recourse to positive realness; IEEE Trans. on Automatic Control, Vol. 49, No. 10, pp. 1762-1767 (2004)

[6] M. Nilsson and B. Egardt: Adaptive tracking for linear plants under fixed feedback; European Journal of 
Control, Vol. 18, No. 5, pp. 411-421 (2012)

[7] M. Eguchi, Q. Mengxing, H. Ohmori, Y. Yamasaki and S. Kaneko: Diesel engine combustion control using feedback error learning with artificial intelligence feedforward controller; Transactions of Society of Automotive Engineers of Japan, Vol. 49, No. 2, pp. 230-234 (2018) (in Japanese)

[8] E. S. Widayaka and H. Ohmori: Combustion control of diesel engine using feedback error learning with Kernel online learning approach; MOVIC2016 Es RASD2016, Journal of Physics: Conference Series 744 (2016) doi:10.1088/1742-6596/744/1/012107

[9] Y. Nakamura, K. Morimoto and S. Wakui: Improvement of auto-tuning for stage positioning with feedback error learning control; Transactions of the JSME (Japan Society of Mechanical Engineering), Vol. 78, No. 795, pp. 108-117 (2012) (in Japanese)

[10] M. Noguchi and K. Sugimoto: Feedback error learning by means of the pre-filter state; Transactions of The Institute of Systems, Control and Information Engineers (ISCIE), Vol. 23, No. 5, pp. 105-110 (2010) (in Japanese)

[11] K. Sugimoto and M. Noguchi: Further results on feedback error learning control: Partially known plant and general response model; 49th IEEE Conference on Decision and Control, pp. 66-71 (2010)

[12] K. Sugimoto and L. A. Mateo: Feedforward learning control for MIMO plant with finite zeros: Parameterization of numerator polynomial matrix; 53rd IEEE Conference on Decision and Control, pp. 4119-4124 (2014)

[13] W. A. Wolovich: Linear Multivariable Systems,
Springer-Verlag (1974)

[14] T. Kailath: Linear Systems, Prentice-Hall (1980)

[15] H. H. Rosenbrock: State Space and Multivariable Theory, Wiley-Interscience (1970)

\section{Authors}

Kenji Sugimoto (Member)

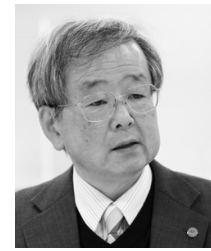

Kenji Sugimoto graduated from Kyoto University in 1980 and received Master's and Doctor's degrees respectively in 1982 and 1989. After working for Mitsubishi Electric Corporation, he was Assistant Professor at Kyoto University, and Associate Professors at Okayama and Nagoya Universities. Currently he is Professor at Nara Institute of Science and Technology. His research interest includes control theory and its application. He is a fellow of SICE and a member of IEEE.

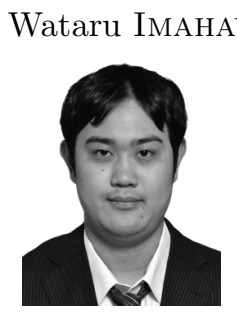

Wataru ImAHAYASHI received B.E degree from Osaka Institute of Technology in 2016 and M.E degree from Nara Institute of Science and Technology in 2018. Currently he is Ph.D student in Nara Institute of Science and Technology. His research interest includes control theory and its application. He is a student member of SICE and IEEE. 